\title{
An Inverse Analysis of the Erichsen Test Applied for the Automatic Identification of Sheet Materials Behavior
}

\author{
Adinel Gavrus ${ }^{1}$, Mihaela Banu ${ }^{2}$, Eric Ragneau ${ }^{1}$, Catalina Maier ${ }^{2}$ \\ ${ }^{1}$ Civil and Mechanical Engineering Laboratory, INSA de RENNES, UEB, Rennes, France \\ ${ }^{2}$ Department of Mechanical Engineering, University “Dunarea de Jos”, UGAL, Galati, Romania \\ E-mail:agavrus@insa-rennes.fr,mihaela.banu@ugal.ro,eric.ragneau@insa-rennes.fr,catalina.maier@ugal.ro \\ Received March 16, 2010; revised March 16, 2010; accepted May 18, 2010
}

\begin{abstract}
Among the technological tests, the Erichsen drawing test gives a more appropriate material behavior, near the limit of the real manufactured process. In this paper an inverse finite element analysis of the Erichsen test is proposed. The new idea is to use a numerical simulation of the experimental test for the rheological identification of the constitutive equations available for sheet metals alloys. The inverse analysis is based on a robust optimization algorithm and uses simultaneously the experimental test data and the corresponding numerical one. A numerical inverse analysis software named OPTPAR was developed and improved for an automatically coupling with a commercial finite element code charged to simulate the experimental test. Results obtained for a virtual steel alloy will be analyzed numerically in order to validate the finite element model and the identification method. An application to an AA5182 aluminum alloy and a DC03 steel alloy will be presented.
\end{abstract}

Keywords: Drawing Process, Finite Element Model, Inverse Analysis, Erichsen Test

\section{Introduction}

Nowadays, the occurrence of the new metallic materials with improved properties destined to the automotive parts generates the computation developments in order to a better identification of their mechanical and technological characteristics. Sheet metal forming is a wide used process in the car panel manufacturing where the formability of the metal sheet has a great importance in prediction of the capacity of the material to be drawn without failure [1]. The occurrence of the new sheet metals like high strength steels, aluminum alloys or nano structured materials with the main target of improvement of the mechanical properties, exhibits new technological challenges. In this context, rapid algorithms of identification of the mechanical and technological behavior are needed to gain time in the competition of producing the parts [2]. Unfortunately the whole complexity of the process requires using numerical modeling to optimize the forming conditions [3-6]. These numerical simulations require available constitutive equations and accurate rheological parameters values, which characterize the sheet material behavior. In order to identify the rheological behavior of the material, the most used test is the uniaxial tensile one and the parameters values are obtained approximately by an analytical analysis using two important hypothesis: the homogeneity of the strain and the small influence of the necking area [7]. In this case the stress-strain curve is available only for small plastic deformations. Moreover, the problem is that the deformation history during the drawing process is different as compared to the uniaxial tensile one, and the constitutive equation can not be available for more severe deformation conditions: strain localization, biaxial expansion or more pronounced localized plastic strain values. In the past decades a preliminary investigation of the metal sheet behavior during drawing process has been possible via the Erichsen or Olsen test [8-9]. Actually this test is standardized and it is used to differentiate different metallic materials used in sheet forming and hydroforming, cold rolling, tube-sinking or incremental sheet forming. In fact the Erichsen test can be used as an indicator of material formability through the height of the spherical punch that deform the blank until the fracture of the material corresponding to a crack of a length approximately equal to $5 \mathrm{~mm}$. In [10] the authors has recently developed a numerical and experimental analysis of Erichsen test via a finite element simulation, but 
only the Erichsen index and the maximal values of the punch force are analyzed. Moreover, several studies based on the behavior analysis of the metallic plate or sheet subjected to a perpendicular impact with a hemispherical projectile [11], show the necessity to explore the material behavior in conditions close to a biaxial expansion, generally developed during the Erichsen test. Consequently, the present paper proposes an application of the inverse analysis principle [12] to the constitutive parameters identification directly from the Erichsen experimental device. The experimental measurements are represented by the recordings of the evolution of the drawing force with the depth of the sheet. The identification of the material coefficients is performed with a finite element simulation of the Erichsen test via an optimization procedure. The corresponding cost function is expressed in terms of the experimental and numerical loads using a least squares formulation. In order to validate the proposed method, this paper neglects the sheet anisotropy (i.e. choice of materials without any anisotropy effect) and the loads are supposed to be only quasi-static ( $i$. $e$. no strain rate sensitivity). Numerical analysis and real results will be presented using the identification high board named OPTPAR (developed in the LGCGM laboratory), automatically coupled with a commercial finite element code (FORGE2 ${ }^{\circledR}$ or MARC one [13]) charged to simulate the Erichsen test.

\section{The Erichsen Experimental Test}

The design of the classical Erichsen test used in the Europe is defined by a hemispherical punch of steel with the diameter of $20 \mathrm{~mm}$, an active die with the diameter of $27 \mathrm{~mm}$, a blank holder with the diameter of $33 \mathrm{~mm}$ and a sheet with the diameter of $90 \mathrm{~mm}$ (see Figure 1(a) and Figure 1(b)). The radius of the die is $0.75 \mathrm{~mm}$.

The hemispherical punch is pressed into the sheet until material fracture occurs, at which point the test is stopped immediately and the depth of the bulge recorded. This depth expressed in millimetres gives the Erichsen index (IE) and obviously gives a measure of the ductility of the sheet under biaxial stress conditions in the plane of the drawing. In a pure stretching forming the sheet is deformed by the punch and is totally clamped. From a classical point of view this test can be used only for comparative purposes of sheet metals. Actually, measurement of the all axial punch force variation can be considered as new potential experimental data. Until now it is not possible to develop a rigorous analytical theory of Erichsen test able to express the punch force in function of the test geometry and of the rheological parameters of the sheet material. It is the principal reason for each a numerical finite element model it is proposed to be used.

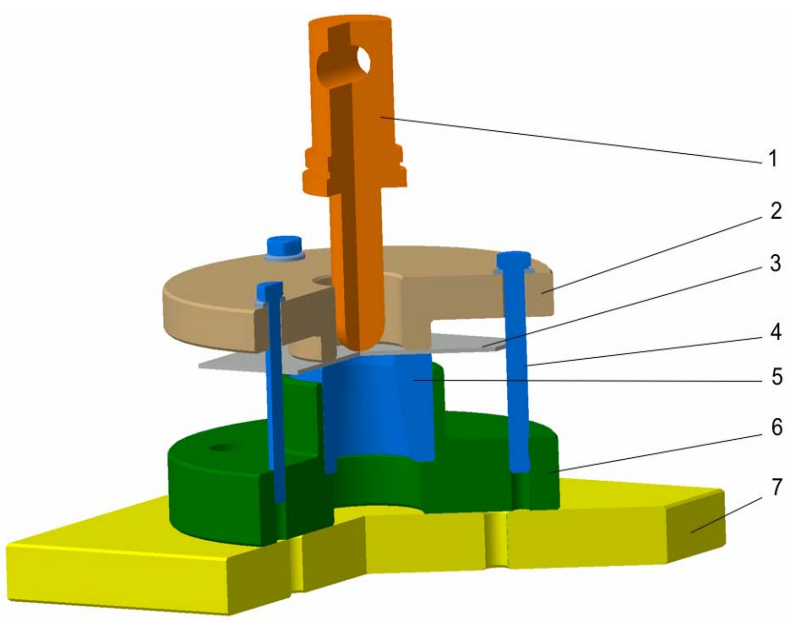

(a)

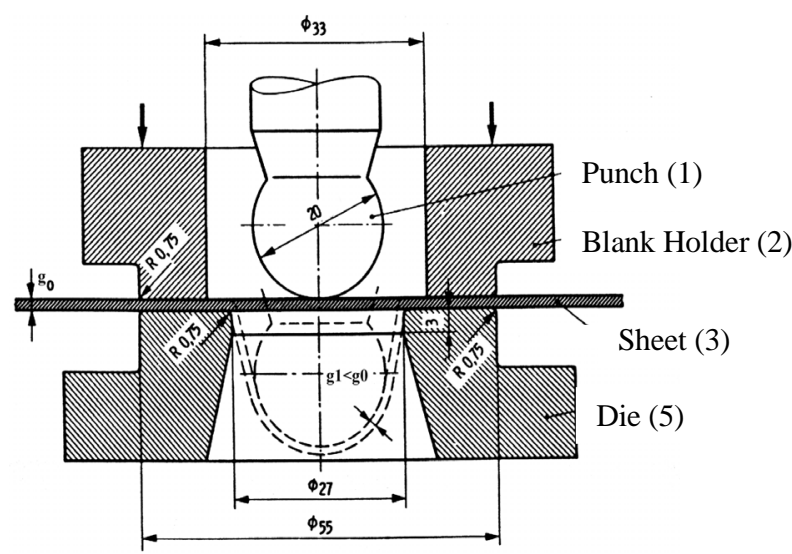

(b)

Figure 1. Experimental set-up of the Erichsen test: (a) 3D view (4, 6, 7-assembly components); (b) 2D asymmetric view.

\section{Numerical Modeling and Analysis}

Starting from a finite element model, a blank, with a diameter of $90 \mathrm{~mm}$ and $3 \mathrm{~mm}$ of thickness, is meshed using three nodes linear triangular elements (Figure 2).

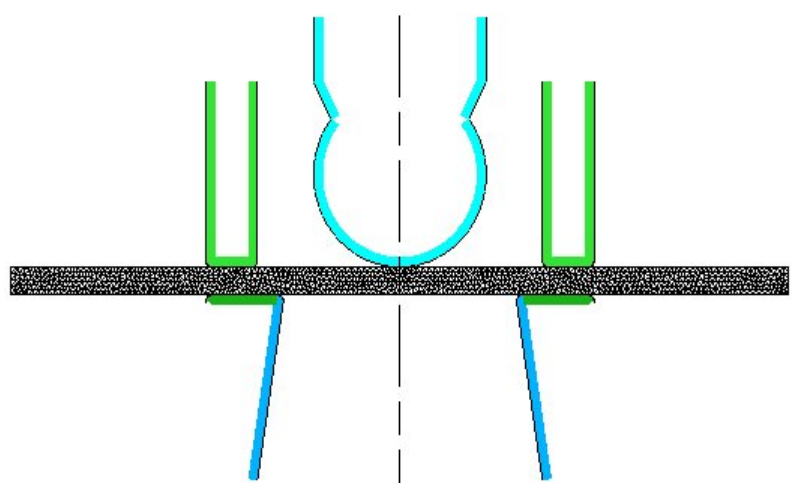

Figure 2. The numerical finite element model. 
Re-meshing procedure is activated during the computation process in order to eliminate numerical problems caused by degenerate elements which can occurs during the numerical simulation. The tools, spherical punch, die and blank holder, are considered to be rigid and the contact between the sheet and the blank holder or the horizontal part of the die is chosen to be a glued one. A Coulomb friction law with a friction coefficient of 0.1 is used between the sheet and the punch or the die active part. Considering an isotropic rheological law of the sheet material we can choose two different descriptions: (1) a Ludwick one and (2) a Voce one (see Figure 3):

$$
\begin{gathered}
\bar{\sigma}=\sigma_{00}+K \bar{\varepsilon}^{n} \\
\bar{\sigma}=\sigma_{00}+K[1-\exp (-n \bar{\varepsilon})]^{n_{a}}
\end{gathered}
$$

where $\sigma_{00}$ is the elastic yield stress, $K$ is the material consistency and $n$ is the hardening parameter. For a steel alloy we must choose $n_{a}=1$.

According to a virtual super plastic and ductile steel alloy, chosen in order to describe more pronounced plastic effects according to a stretching process, the values of the elastic and plastic material data are presented in Table 1.

The numerical simulations have been realized using the FORGE2 commercial code (with an initial number of elements equal to 947) and with the MARC software (with an initial number of elements equal to 346). After $18 \mathrm{~mm}$ of the punch displacement the results of the deformed mesh and of the cumulated plastic strain distribution are pictured in Figure $\mathbf{4}$ and Figure 5.

The similar results obtained with the both numerical codes confirm the availability of the numerical modeling. It is then possible to show that the necking phenomenon occurs at approximately 30 degree under the punch.

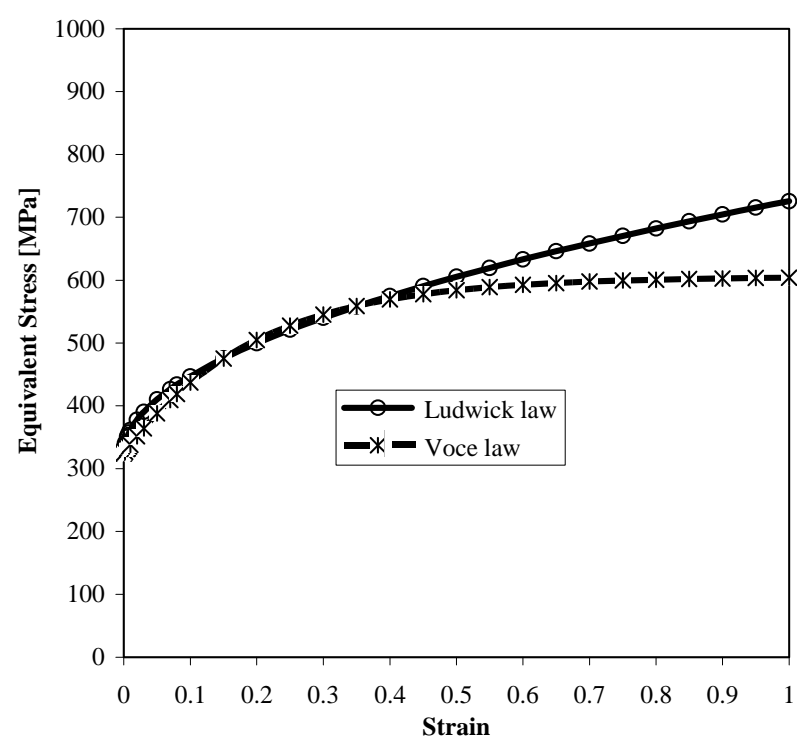

Figure 3. Rheological laws used for the numerical simulation.
Table 1. Material properties for a virtual steel sheet.

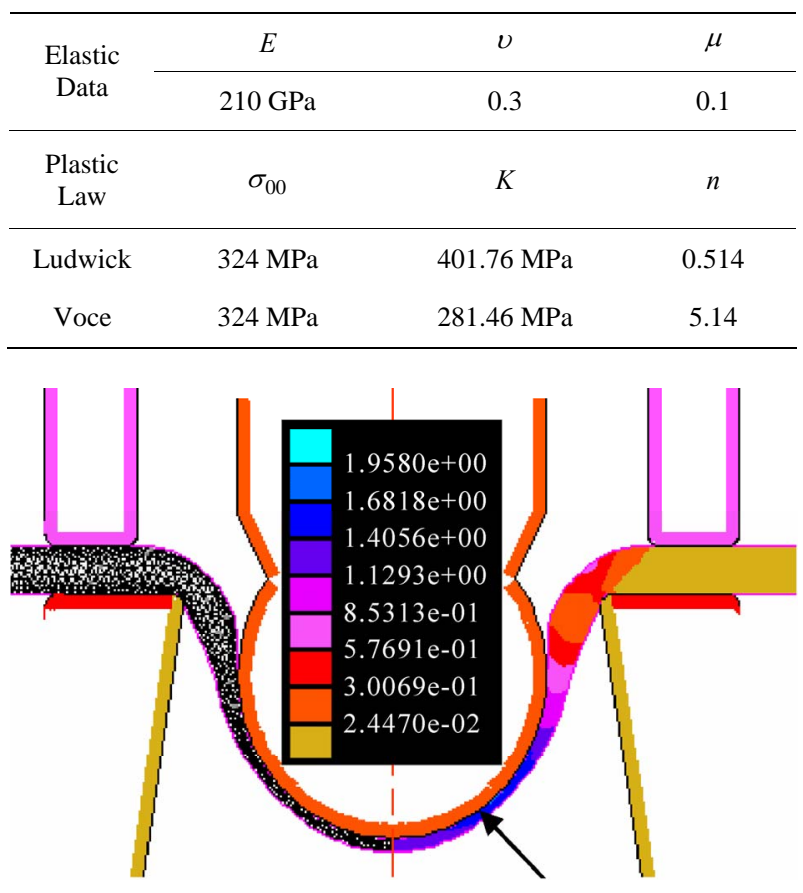

Figure 4. Numerical finite element results obtained with FORGE2 (Mesh and cumulated plastic strain at $t=18 \mathrm{~s}$ ).

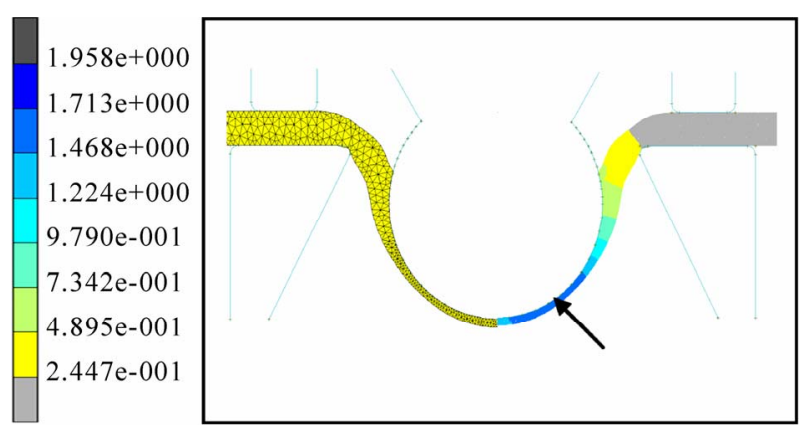

Figure 5. Numerical finite element results obtained with MARC (Mesh and cumulated plastic strain at $t=18 \mathrm{~s}$ ).

Moreover, the obtained large values of the cumulated plastic strain (up to $150 \%$ ) permits a deformation history analysis for more severe numerical conditions, very close to a real stretching or a drawing process of a super plastic alloy. Considering the variation of the axial punch force, the results are plotted in Figure 6. These results show that similar curve shapes are obtained from the FORGE2 code and the MARC one. The small differences are caused by the numerical treatment of the contact phenomena, because different methods are used to adjust numerically the Coulomb law [13].

Concerning the influence of the material rheological law, we obtain a more pronounced softening with the Voce formulation of the stress-strain variation. The use of the extrapolation values for the stress from a classical Ludwick law can causes several problems for the estima- 


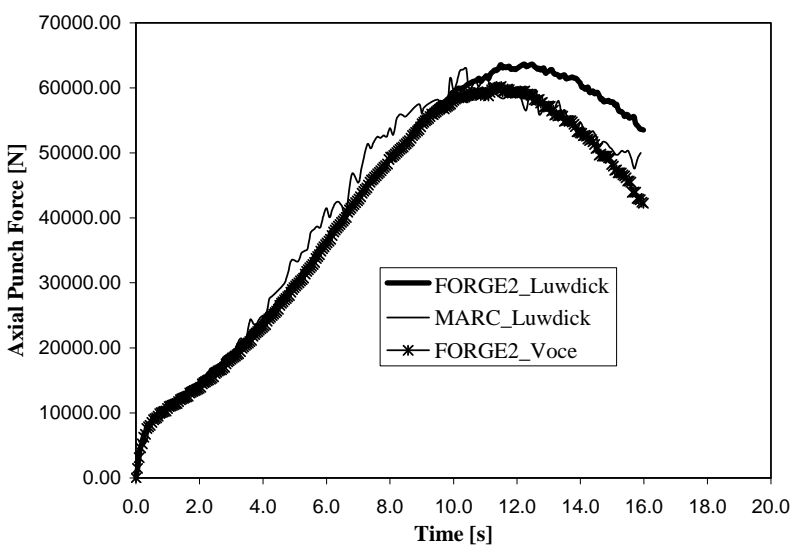

Figure 6. Results of the axial punch force variation.

tion of the process forces or for the computation of the deformation energies.

\section{Validation of the Erichsen Test Simulation for an Aluminum Alloy}

In order to validate the finite element simulation of the Erichsen test, a deformation of an aluminum alloy AA5182 is analyzed. Experimentally, for $7.6 \mathrm{~mm}$ of the punch displacement, the thickness of the sheet in the necking area is approximately of $0.5 \mathrm{~mm}$. The friction phenomenon and Coulomb parameter are considered to be a priori known (i.e. $\mu=0.1$ ) and in order to eliminate the anisotropy of aluminum alloys, a special thermal treatment has been used. So the rheological law is described only by a rigid plastic model $(K=0)$ where $\sigma_{00}$ is approximately equal to $74 \mathrm{MPa}$. Particular problems caused by the sensitivity of the sheet deformation with respect to other constitutive parameters (as for example the hardening ones) are then avoided. Starting from a numerical finite element simulation via the FORGE2 software, the sheet geometry and the distribution of the plastic strain after $7.6 \mathrm{~mm}$ of the punch displacement is pictured in Figure 7.

Figure 8 shows the fracture of the material obtained from the real experiment. It is important to note that numerically we obtain approximately the same final thickness of the sheet.

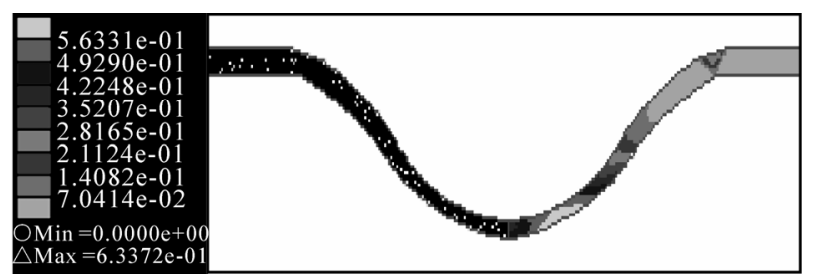

Figure 7. The numerical finite element results obtained from FORGE2 (Mesh and cumulated plastic strain corresponding to $7.6 \mathrm{~mm}$ of the punch displacement).

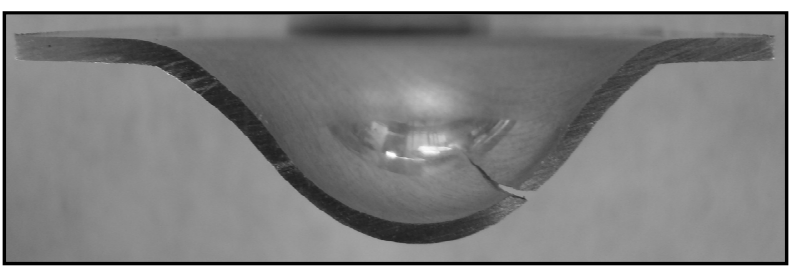

Figure 8. Image of the fracture obtained by experiment (Erichsen index equal to $7.8 \mathrm{~mm}$ ).

The cumulated plastic strain in the necking area is approximately $60 \%$, which corresponds to the analytical estimation of the logarithmic plastic strain obtained from the division between the initial and the final thickness. These results allow us to confirm that large plastic deformations occur during the process and these ones cannot be obtained from a classical tensile test. Many experiments performed in laboratory LGCGM of INSA de RENNES, which compare the cumulated plastic strain values during a biaxial tensile test and a uniaxial tensile one, confirm this last assertion. Finally, complete content and organizational editing before formatting. Please take note of the following items when proofreading spelling and grammar.

\section{Parameter Identification by Inverse Analysis}

Starting from the previous experimental and numerical analysis, accurate values of the constitutive parameters behaviour can be obtained using the inverse analysis principle. The experimental data can be represented by the recordings of the evolution of the punch force with the depth of the sheet (Figure 6). The identification of the material coefficients can be then performed with a finite element simulation of the Erichsen test via an optimization procedure (OPTPAR, see Figure 9). Using the least squares formula the cost function is expressed in terms of the experimental forces and of the numerical ones by:

$$
\Phi\left(P, F^{c}, F^{\exp }\right)=\sum_{i=1}^{N \text { exp }}\left[F_{i}^{c}-F_{i}^{\exp }\right]^{2} / \sum_{i=1}^{N \exp }\left[F_{i}^{\exp }\right]^{2}
$$

where $P$ is the parameter vector: $P=\{K, n\}, N \exp$ is the number of the experimental points, $F c$ is the finite element computed punch forces and the $F \exp$ is the corresponding experimental data.

For a rheological identification problem, an appropriate physical domain of parameters variation must be introduced and a constrained optimization problem must be formulated as:

$$
\left\{\begin{array}{l}
\min _{P \in D(P)} \Phi\left(P, F^{c}, F^{\text {exp }}\right) \\
D(P)=\left\{P / P_{\text {min }} \leq P \leq P_{\text {max }}\right\}
\end{array}\right.
$$




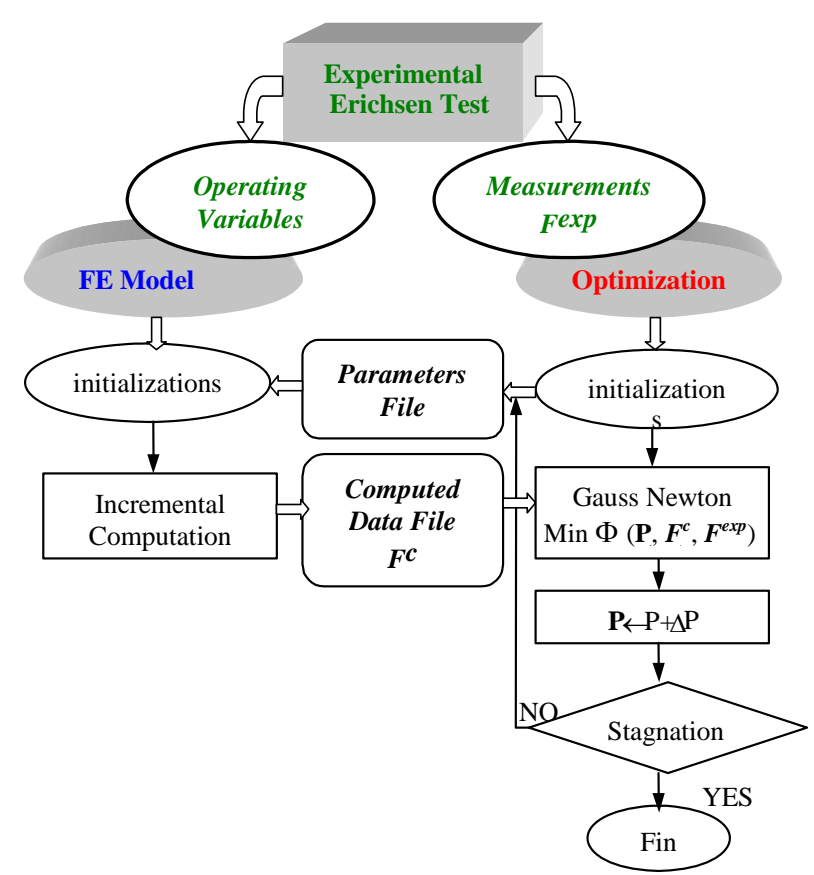

Figure 9. Algorithm of the inverse analysis method.

The high no linearity of the objective function requires a robust numerical minimization algorithm based on the Gauss-Newton gradient method. In this case evaluation of the first and second objective function derivatives are required:

$$
\begin{aligned}
& \frac{d \Phi}{d P_{j}}=\frac{2 \sum_{i=1}^{N \exp }\left[\frac{d F_{i}^{c}}{d P_{j}}\right]\left[F_{i}^{c}-F^{\exp }\right]}{\sum_{i=1}^{N \exp }\left[F_{i}^{\exp }\right]^{2}} \\
& \frac{d^{2} \Phi}{d P_{j k}} \approx \frac{2 \sum_{i=1}^{N \exp }\left[\frac{d F_{i}^{c}}{d P_{j}}\right]\left[\frac{d F_{i}^{c}}{d P_{k}}\right]}{\sum_{i=1}^{N \exp }\left[F_{i}^{\exp }\right]^{2}}
\end{aligned}
$$

A direct differentiation method is used to compute the derivatives of the computed forces with respect to the constitutive parameters. This method requires adding numerical simulations corresponding to very small variations of each parameter, which will be identified. To test the numerical convergences of the inverse analysis procedure, an artificial experimental data is used starting from supposed known material parameters presented in Table 1. The goal of convergence analysis is to verify the capacity of the identification algorithm to find the true rheological parameters, starting from different initial estimations. The optimization procedure is initiated for both laws, using initial values sufficiently far from the real ones (see Table 2 and Table 3).
Table 2. Numerical identified results for the first initial guess values.

\begin{tabular}{ccccc}
\hline \multirow{2}{*}{ Parameters } & \multicolumn{2}{c}{ Ludwick Law } & \multicolumn{2}{c}{ Voce Law } \\
\cline { 2 - 5 } & Initial & Identif. & Initial & Identif. \\
\hline$\sigma_{00}$ & 324. & 324. & 324. & 324. \\
$K[\mathrm{MPa}]$ & 500. & 401.79 & 500. & 281.47 \\
$n$ & 0.25 & 0.514 & 1. & 5.14 \\
$\Phi$ & $22.63 \%$ & $0.008 \%$ & $11.03 \%$ & $0.003 \%$ \\
iterations & - & 15 & - & 13 \\
\hline
\end{tabular}

Table 3. Numerical identified results for the second initial guess values.

\begin{tabular}{ccccc}
\hline \multirow{2}{*}{ Parameters } & \multicolumn{2}{c}{ Ludwick Law } & \multicolumn{2}{c}{ Voce Law } \\
\cline { 2 - 5 } & Initial & Identif. & Initial & Identif. \\
\hline$\sigma_{00}$ & 324. & 324. & 324. & 324. \\
$K$ & 1000. & 401.74 & 100. & 281.38 \\
$n$ & 0.1 & 0.514 & 10. & 5.147 \\
$\Phi$ & $103 \%$ & $0.004 \%$ & $27 \%$ & $0.07 \%$ \\
iterations & - & 11 & - & 9 \\
\hline
\end{tabular}

These results show that the numerical convergence is obtained in a small number of optimization iterations and with a very high precision (objective function smallest that $0.1 \%$ ). The available numerical inverse analysis model can be now used in a real experimental test.

\section{Application to a Real Data}

In order to test the inverse analysis of the Erichsen test in real conditions, a rheological parameter identification is made for a DC03 steel alloy and using a sheet with the initial thickness equal to $1 \mathrm{~mm}$.

The axial punch force is recorded until the material fracture occurs. The friction law is supposed to be a Coulomb one and the friction parameter is a priori known $(\mu=0.1)$. For the numerical model, a mesh with approximately 853 elements is used. The Table 4 synthesis the parameter identification results obtained with the OPTPAR software. These results show that the material has, for the Ludwick law, a normal hardening effect $(n=0.61)$ and for the Voce law a small value of the Voce parameter $(n=2.47)$. The comparison between the experimental and the computed loads is plotted in Figure 10.

The global error is approximately $1 \%$ for the Ludwick law and $3 \%$ for the Voce one and it is easy to see that the computed load curves are very close to the experimental ones. It is then possible to conclude that the constitutive parameters defining the equivalent Von-Mises stress can be obtained directly from the Erichsen experimental test. 
Table 4. Numerical parameter identification results for a real DC03 steel sheet.

\begin{tabular}{ccccc}
\hline \multirow{2}{*}{ Parameters } & \multicolumn{2}{c}{ Ludwick Law } & \multicolumn{2}{c}{ Voce Law $\left(n_{a}=1\right)$} \\
\cline { 2 - 5 } & Initial & Identified & Initial & Identified \\
\hline$\sigma_{00}$ & 150. & 154.17 & 154.17 & 154.17 \\
$K[\mathrm{MPa}]$ & 500. & 516.52 & 500. & 484.33 \\
$n$ & 0.25 & 0.61 & 10. & 2.47 \\
$\Phi$ & $19.8 \%$ & $1 \%$ & $34.7 \%$ & $2.7 \%$ \\
iterations & - & 6 & - & 4 \\
\hline
\end{tabular}

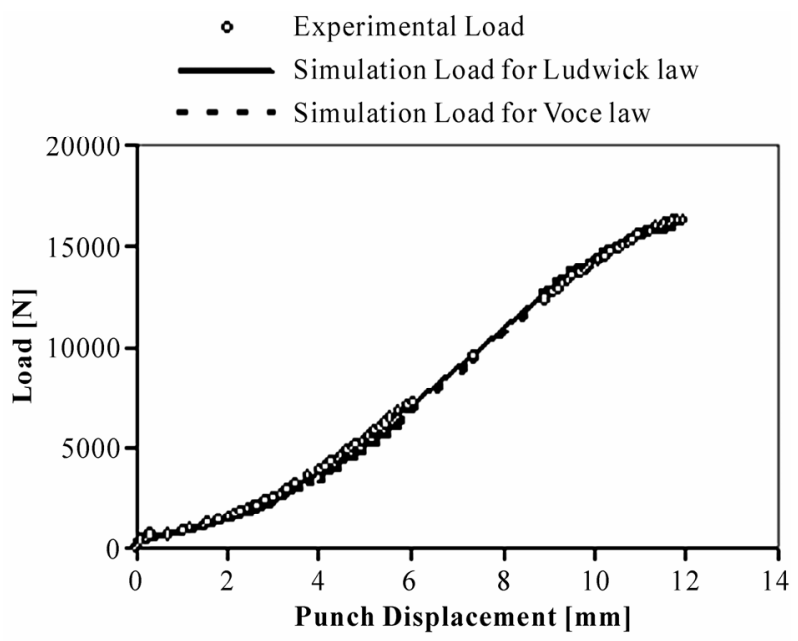

Figure 10. Comparison between experimental and computed axial punch forces corresponding to the previous identified rheological laws.

\section{Conclusions}

A robust optimization algorithm for the constitutive parameters identification was developed from a numerical simulation of the Erichsen test. This inverse method uses the commercial codes FORGE2 and MARC one starting only from the variation of the axial punch force. The inverse analyses, combined with numerical validations, robustness studies and validation through a real experiment, underlines the accuracy of the results obtained by proposed algorithm. The main feature of this identification methodology is linked to the possibility to take into account the all complexity of the test. The finite element model permits to simulate the biaxial stress conditions, together with a non-uniform distribution of the plastic strain and of the sheet thickness. It is then possible to obtain deformation conditions close to those which occur during a real drawing or stretching process. In a future work, complementary experimental data, as for example the angular position of the necking radius together with the values of the sheet thickness variation, will be added.

\section{Acknowledgements}

The present work was elaborated under the bilateral research agreement established between INSA de RENNES-France and University "Dunarea de Jos" GALATIRomania (UGAL), and financially supported by the research contracts CNCSIS 686/2007 and CEEX 24/2006 of the laboratory ITCM of UGAL.

\section{References}

[1] D. Banabic, "Formability of Metallic Materials (Plastic Anisotropy, Formability Testing and Forming Limits)," Springer Verlag, Berlin, Germany, 2000.

[2] R. Padmanabhan, M. C. Oliveir, J. L. Alves and L. F. Menezes, "Influence of Process Parameters on the Deep Drawing of Stainless Steel," Finite Elements in Analysis and Design Archive, Vol. 43, No. 14, 2007, pp. 1062-1067.

[3] J.-L. Batoz, H. Naceur and Y.-O. Guo, "Sheet Metal Stamping Analysis and Process Design Based on the Inverse Approach," Proceeding of 10th Esaform Conference on Materials Forming, Zaragozza, Spain, 2007, pp. 1448-1453.

[4] H. Naceur, A. Delamezier, J. L. Batoz, Y. O. Guo and C. $\mathrm{K}$. Lenoir, "Some Improvements on the Optimum Process Design in Deep Drawing Using the Inverse Approach,” Journal of Materials Processing Techenology, Vol. 146, No. 2, 2004, pp. 250-262.

[5] V. Paunoiu and D. Nicoara, "Simulation of Friction Phenomenon in Deep Drawing Process," The Annals of University "Dunărea de Jos" of Galați Fascicle VIII, Tribology, 2003, pp. 407-412.

[6] R. J. Comstock, K. Li and R. H. Wagoner, "Simulation of Axisymmetric Sheet Forming Tests," Journal of Materials Processing Techenology, Vol. 117, No. 1, 2001, pp. 153-168.

[7] W. G. Granzou, “Sheet Formability of Steel,” 10th Edition, ASM International, Metals Handbook, Materials Park, Ohio, 1990.

[8] B. Kaftanoglu and J. M. Alexander, "An Investigation of the Erichsen Test," Journal of the Institute of Metals, Vol. 90, 1961, pp. 457- 470.

[9] T. Y. Olsen, "Machines for Ductility Testing," Proceeding of the American Society of Materials, Vol. 20, 1920, pp. 398-403.

[10] M. Akrout, M. B. Amar, C. Chaker and F. Dammak, "Numerical and Experimental Study of the Erichsen Test for Metal Stamping," Advances in Production Engineering \& Management, Vol. 3, No. 2, 2008, pp. 81-92.

[11] A. Gavrus, V. Grolleau and S. Diot, "Experimental and Numerical Analysis of an Impacted Thin Aluminum Plate," Proceedings of Structures Under Shock and Impact VII, Montreal, 2002, pp. 477-486.

[12] A. Gavrus, "Identification Des Parametres Rheologiques Par Analyse Inverse,” Phd Thesis, ENSMP, Paris, 1997.

[13] MARC User's Manual, Analysis Research Corporation, 1997. 